\title{
In silico study on $\beta$-aminoketone derivatives as thyroid hormone receptor inhibitors: a combined 3D-QSAR and molecular docking study
}

\section{Fang-Fang Wang ${ }^{\mathrm{a}}$, Wei Yang ${ }^{\mathrm{b}}$, Yong-Hui Shic, Guo-Wei Le ${ }^{\mathrm{a}, \mathrm{c}, *}$}

${ }^{a}$ The State Key Laboratory of Food Science and Technology, Jiangnan University, Wuxi, 214122, China.

${ }^{\mathrm{b}}$ Department of Biochemistry and Molecular Biology, Faculty of Medicine, Monash University, Melbourne, VIC 3800, Australia.

${ }^{\mathrm{c}}$ The Institution of Food Nutrition and Safety, School of Food Science and Technology, Jiangnan University, Wuxi, 214122, China.

* Corresponding author: Tel.: +86-510-8591-7789; Fax: + 86-510-8591-7789.

E-mail: lgw@jiangnan.edu.cn

Table S1. Molecular structures of Indane Derivatives and their TR $\beta$ binding affinity values $\left(\mathrm{pIC}_{50}\right)$.

\begin{tabular}{|c|c|c|c|}
\hline Compound & Structure & TRß1 pKi & TRa1 pK \\
\hline 1 & & 4.7825 & - \\
\hline 2 & & 5.1487 & - \\
\hline 3 & & 5.3979 & - \\
\hline 4 & & 5.7696 & - \\
\hline 5 & & 5.6383 & - \\
\hline 6 & & 5.7959 & - \\
\hline 7 & & 5.5686 & - \\
\hline
\end{tabular}




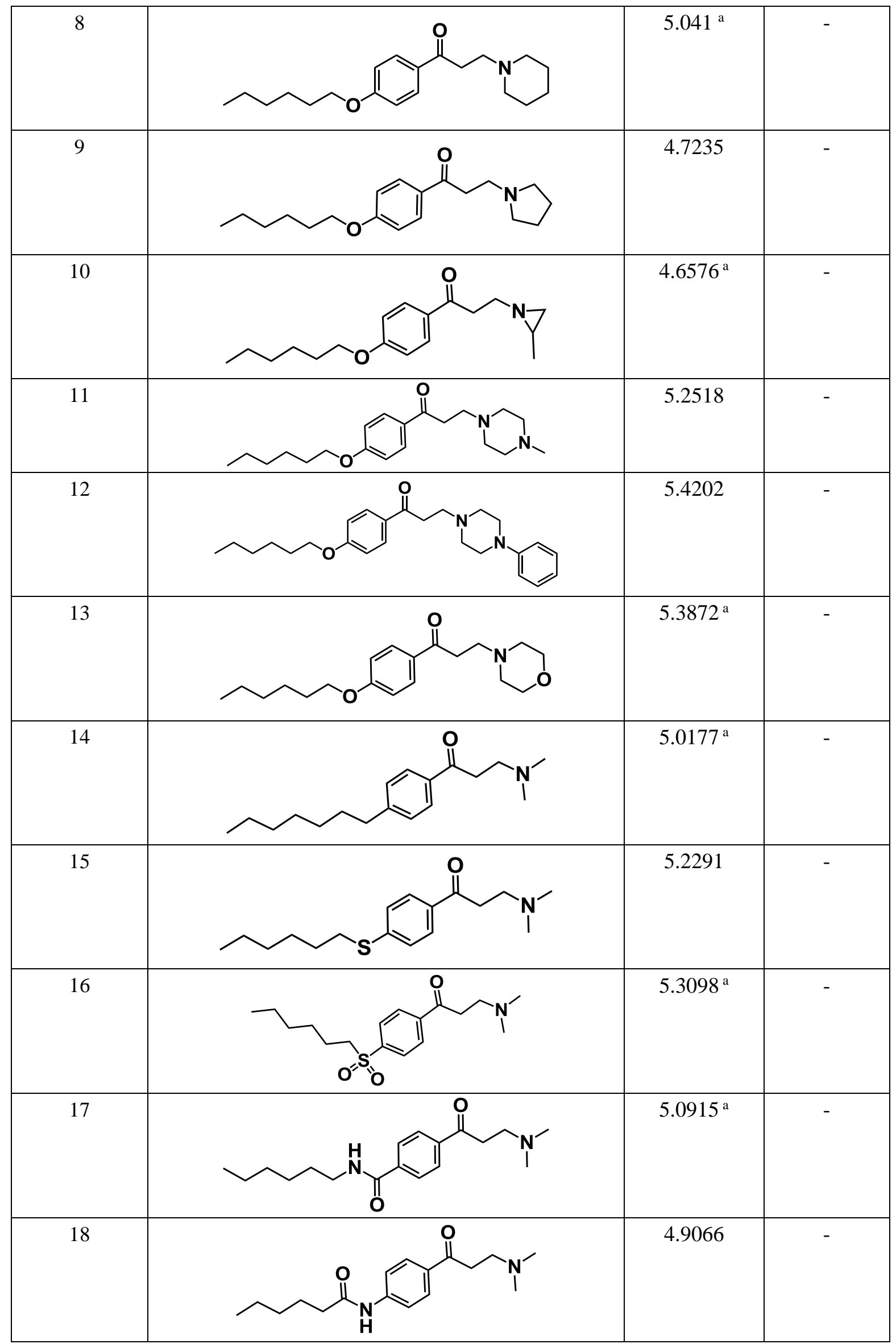




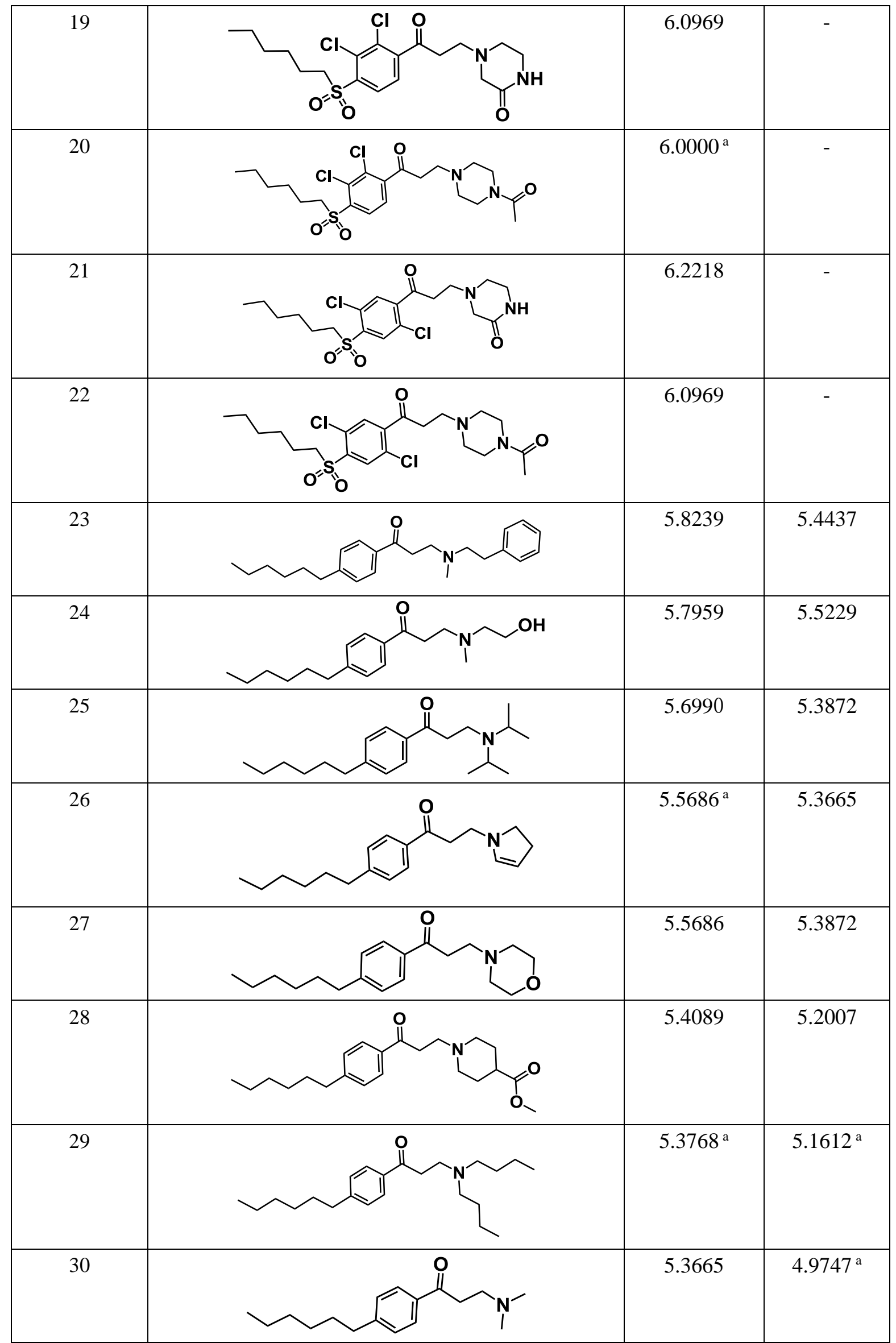




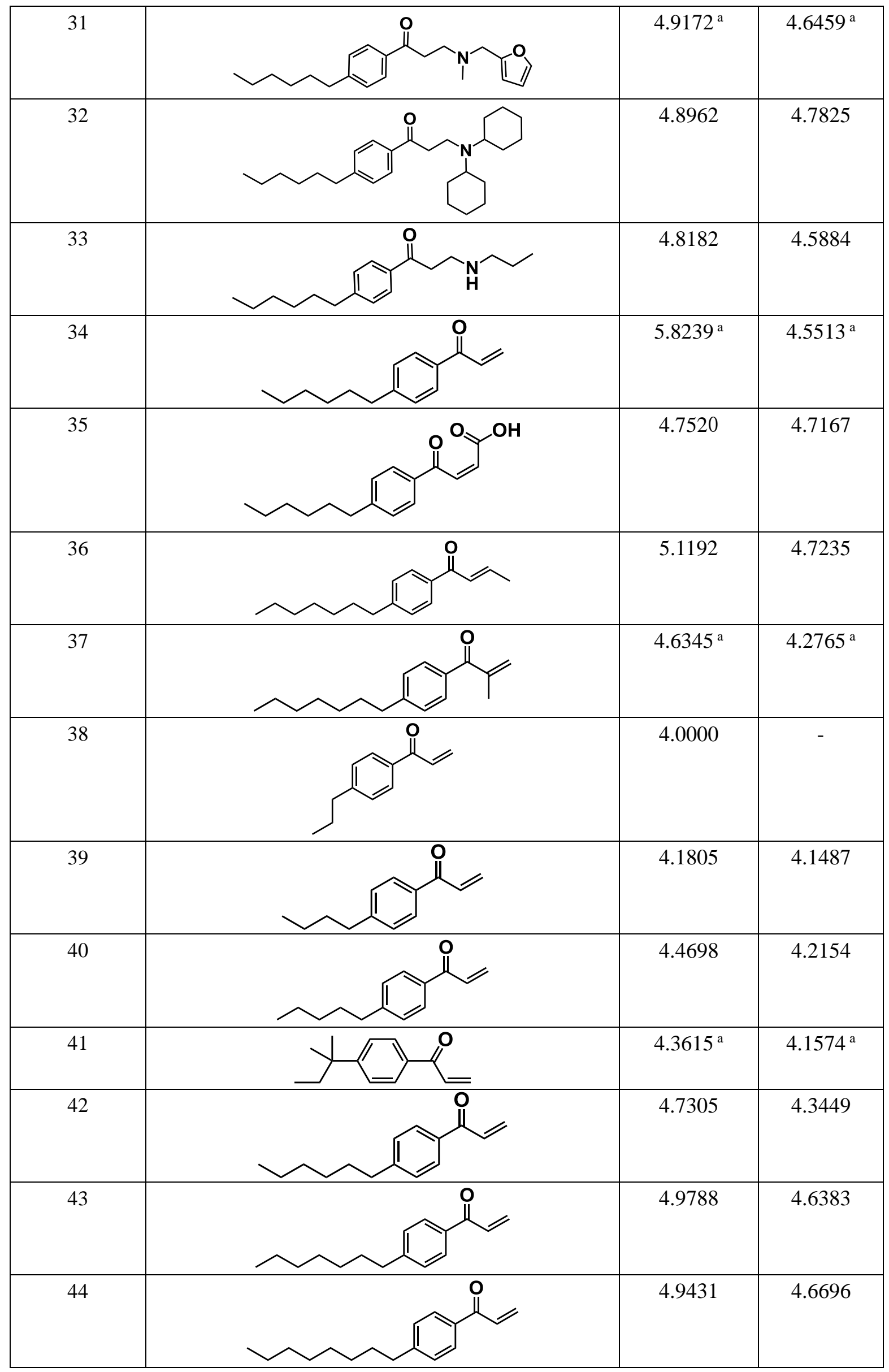




\begin{tabular}{|l|l|l|l|}
\hline & & \\
\hline
\end{tabular}


${ }^{a}$ represents the test set.

Table S2. Summary of QSAR results for TR $\beta$ based on superimposition I .

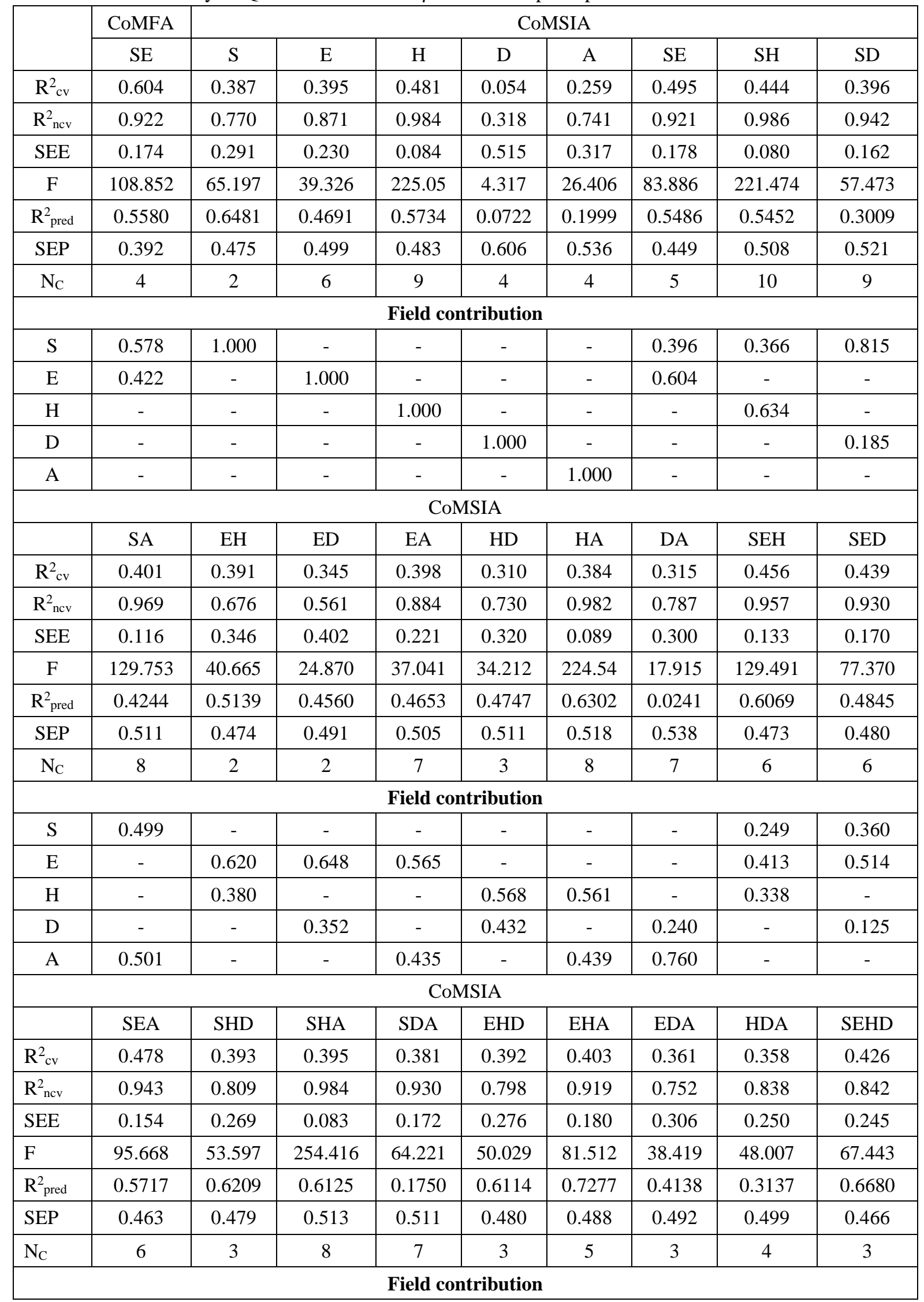




\begin{tabular}{|c|c|c|c|c|c|c|c|c|c|}
\hline$S$ & 0.283 & 0.256 & 0.253 & 0.399 & - & - & - & - & 0.188 \\
\hline E & 0.412 & - & - & - & 0.373 & 0.355 & 0.404 & - & 0.306 \\
\hline $\mathrm{H}$ & - & 0.404 & 0.393 & - & 0.353 & 0.343 & - & 0.391 & 0.276 \\
\hline $\mathrm{D}$ & - & 0.340 & - & 0.181 & 0.274 & - & 0.271 & 0.261 & 0.230 \\
\hline $\mathrm{A}$ & 0.305 & - & 0.354 & 0.420 & - & 0.302 & 0.325 & 0.348 & - \\
\hline \multicolumn{10}{|c|}{ CoMSIA } \\
\hline & \multicolumn{2}{|c|}{ SEHA } & SEDA & \multicolumn{2}{|c|}{ SHDA } & \multicolumn{2}{|c|}{ EHDA } & \multicolumn{2}{|c|}{ SEHDA } \\
\hline $\mathrm{R}_{\mathrm{cv}}^{2}$ & \multicolumn{2}{|c|}{0.447} & 0.435 & \multicolumn{2}{|r|}{0.394} & \multicolumn{2}{|c|}{0.391} & \multicolumn{2}{|c|}{0.413} \\
\hline $\mathrm{R}_{\text {ncv }}^{2}$ & \multicolumn{2}{|c|}{0.969} & 0.897 & \multicolumn{2}{|r|}{0.870} & \multicolumn{2}{|r|}{0.846} & \multicolumn{2}{|c|}{0.832} \\
\hline SEE & \multicolumn{2}{|c|}{0.115} & 0.203 & \multicolumn{2}{|r|}{0.225} & \multicolumn{2}{|r|}{0.244} & \multicolumn{2}{|c|}{0.252} \\
\hline $\mathrm{F}$ & \multicolumn{2}{|c|}{151.282} & 62.523 & \multicolumn{2}{|r|}{61.959} & \multicolumn{2}{|c|}{50.871} & \multicolumn{2}{|c|}{62.786} \\
\hline $\mathrm{R}_{\text {pred }}^{2}$ & \multicolumn{2}{|c|}{0.6599} & 0.4685 & \multicolumn{2}{|r|}{0.3728} & \multicolumn{2}{|c|}{0.5946} & \multicolumn{2}{|c|}{0.6330} \\
\hline SEP & \multicolumn{2}{|c|}{0.484} & 0.475 & \multicolumn{2}{|r|}{0.485} & \multicolumn{2}{|c|}{0.486} & \multicolumn{2}{|c|}{0.471} \\
\hline $\mathrm{N}_{\mathrm{C}}$ & \multicolumn{2}{|c|}{7} & 5 & & 4 & \multicolumn{2}{|r|}{4} & \multicolumn{2}{|c|}{3} \\
\hline \multicolumn{10}{|c|}{ Field contribution } \\
\hline $\mathrm{S}$ & \multicolumn{2}{|c|}{0.191} & 0.235 & & 0.196 & & - & & 147 \\
\hline E & 0.301 & & 0.355 & & - & & 0.285 & & 245 \\
\hline $\mathrm{H}$ & 0.276 & & - & & 0.297 & & 0.278 & & 214 \\
\hline $\mathrm{D}$ & - & & 0.141 & & 0.233 & & 0.198 & & 198 \\
\hline A & 0.232 & & 0.268 & & 0.274 & & 0.240 & & 197 \\
\hline
\end{tabular}

Table S3. Summary of QSAR results for TR $\beta$ based on superimposition II (the lowest binding

\begin{tabular}{|c|c|c|c|c|c|c|c|c|c|}
\hline & CoMFA & \multicolumn{8}{|c|}{ CoMSIA } \\
\hline & SE & $S$ & $\mathrm{E}$ & $\mathrm{H}$ & $\mathrm{D}$ & A & SE & $\mathrm{SH}$ & SD \\
\hline $\mathrm{R}_{\mathrm{cv}}^{2}$ & 0.649 & 0.657 & 0.611 & 0.611 & 0.619 & 0.609 & 0.666 & 0.623 & 0.629 \\
\hline $\mathrm{R}_{\mathrm{ncv}}^{2}$ & 0.994 & 0.787 & 0.654 & 0.650 & 0.660 & 0.651 & 0.922 & 0.920 & 0.882 \\
\hline SEE & 0.051 & 0.280 & 0.352 & 0.355 & 0.349 & 0.354 & 0.172 & 0.174 & 0.215 \\
\hline $\mathrm{F}$ & 925.504 & 72.216 & 75.758 & 74.349 & 77.654 & 74.566 & 149.043 & 144.835 & 68.825 \\
\hline $\mathrm{R}_{\text {pred }}^{2}$ & 0.6081 & 0.6963 & 0.7666 & 0.7717 & 0.7690 & 0.7637 & 0.6219 & 0.7182 & 0.6680 \\
\hline SEP & 0.380 & 0.356 & 0.374 & 0.374 & 0.370 & 0.375 & 0.356 & 0.378 & 0.380 \\
\hline $\mathrm{N}_{\mathrm{C}}$ & 6 & 2 & 1 & 1 & 1 & 1 & 3 & 3 & 4 \\
\hline \multicolumn{10}{|c|}{ Field contribution } \\
\hline$S$ & 0.394 & 0.549 & - & - & - & - & 0.301 & 0.322 & 0.450 \\
\hline $\mathrm{E}$ & 0.439 & - & 0.147 & - & - & - & 0.433 & - & - \\
\hline $\mathrm{H}$ & - & - & - & 0.120 & - & - & - & 0.409 & - \\
\hline $\mathrm{D}$ & - & - & - & - & 0.180 & - & - & - & 0.262 \\
\hline $\mathrm{A}$ & - & - & - & - & - & 0.136 & - & - & - \\
\hline EEig06d & 0.167 & 0.451 & 0.853 & 0.880 & 0.820 & 0.864 & 0.266 & 0.269 & 0.287 \\
\hline \multicolumn{10}{|c|}{ CoMSIA } \\
\hline & SA & EH & ED & EA & HD & HA & DA & SEH & SED \\
\hline $\mathrm{R}_{\mathrm{cv}}^{2}$ & 0.612 & 0.626 & 0.611 & 0.601 & 0.611 & 0.602 & 0.609 & 0.661 & 0.656 \\
\hline $\mathrm{R}_{\text {ncv }}^{2}$ & 0.658 & 0.999 & 0.664 & 0.655 & 0.661 & 0.652 & 0.662 & 0.947 & 0.957 \\
\hline
\end{tabular}




\begin{tabular}{|c|c|c|c|c|c|c|c|c|c|}
\hline SEE & 0.350 & 0.018 & 0.347 & 0.352 & 0.349 & 0.354 & 0.349 & 0.141 & 0.130 \\
\hline $\mathrm{F}$ & 77.056 & 432.819 & 79.117 & 75.965 & 78.054 & 74.804 & 78.32 & 227.904 & 204.817 \\
\hline $\mathrm{R}_{\text {pred }}^{2}$ & 0.7622 & 0.6267 & 0.7525 & 0.7473 & 0.7593 & 0.7530 & 0.7516 & 0.6131 & 0.4285 \\
\hline SEP & 0.373 & 0.417 & 0.374 & 0.379 & 0.374 & 0.378 & 0.375 & 0.358 & 0.366 \\
\hline $\mathrm{N}_{\mathrm{C}}$ & 1 & 10 & 1 & 1 & 1 & 1 & 1 & 3 & 4 \\
\hline \multicolumn{10}{|c|}{ Field contribution } \\
\hline $\mathrm{S}$ & 0.063 & - & - & - & - & - & - & 0.197 & 0.232 \\
\hline $\mathrm{E}$ & - & 0.420 & 0.123 & 0.129 & - & - & - & 0.329 & 0.365 \\
\hline $\mathrm{H}$ & - & 0.360 & - & - & 0.101 & 0.105 & - & 0.229 & - \\
\hline $\mathrm{D}$ & - & - & 0.158 & - & 0.162 & - & 0.160 & - & 0.193 \\
\hline A & 0.127 & - & - & 0.118 & - & 0.121 & 0.114 & - & - \\
\hline EEig06d & 0.810 & 0.220 & 0.719 & 0.753 & 0.737 & 0.773 & 0.726 & 0.245 & 0.210 \\
\hline \multicolumn{10}{|c|}{ CoMSIA } \\
\hline & SEA & SHD & SHA & SDA & EHD & EHA & EDA & HDA & SEHD \\
\hline $\mathrm{R}_{\mathrm{cv}}^{2}$ & 0.615 & 0.613 & 0.604 & 0.611 & 0.603 & 0.603 & 0.600 & 0.602 & 0.642 \\
\hline $\mathrm{R}_{\text {ncv }}^{2}$ & 0.931 & 0.668 & 0.659 & 0.669 & 0.665 & 0.999 & 0.665 & 0.662 & 0.967 \\
\hline SEE & 0.162 & 0.345 & 0.350 & 0.345 & 0.347 & 0.024 & 0.347 & 0.348 & 0.114 \\
\hline $\mathrm{F}$ & 170.357 & 80.549 & 77.176 & 80.879 & 79.369 & 248.559 & 79.35 & 78.506 & 269.218 \\
\hline $\mathrm{R}_{\text {pred }}^{2}$ & 0.5820 & 0.7576 & 0.7516 & 0.7506 & 0.7438 & 0.5018 & 0.7352 & 0.7425 & 0.4759 \\
\hline SEP & 0.381 & 0.373 & 0.377 & 0.374 & 0.378 & 0.429 & 0.379 & 0.378 & 0.383 \\
\hline $\mathrm{N}_{\mathrm{C}}$ & 3 & 1 & 1 & 1 & 1 & 10 & 1 & 1 & 4 \\
\hline \multicolumn{10}{|c|}{ Field contribution } \\
\hline$S$ & 0.228 & 0.054 & 0.057 & 0.053 & - & - & - & - & 0.170 \\
\hline $\mathrm{E}$ & 0.332 & - & - & - & 0.112 & 0.322 & 0.111 & - & 0.292 \\
\hline $\mathrm{H}$ & - & 0.095 & 0.100 & - & 0.089 & 0.285 & - & 0.090 & 0.174 \\
\hline $\mathrm{D}$ & - & 0.153 & - & 0.151 & 0.144 & - & 0.142 & 0.145 & 0.158 \\
\hline $\mathrm{A}$ & 0.201 & - & 0.115 & 0.108 & - & 0.192 & 0.101 & 0.104 & - \\
\hline EEig06d & 0.239 & 0.697 & 0.729 & 0.687 & 0.654 & 0.201 & 0.646 & 0.661 & 0.206 \\
\hline & & & & $\mathrm{CoN}$ & ISIA & & & & \\
\hline & SEHA & & EDA & $\mathrm{SH}$ & & EHDA & & SEHD & \\
\hline $\mathrm{R}_{\mathrm{cv}}^{2}$ & 0.621 & & 0.602 & 0.6 & & 0.592 & & 0.59 & \\
\hline $\mathrm{R}_{\mathrm{ncv}}^{2}$ & 0.947 & & 0.672 & 0.6 & & 0.665 & & 0.672 & \\
\hline SEE & 0.142 & & 0.343 & 0.3 & & 0.347 & & 0.343 & \\
\hline $\mathrm{F}$ & 225.443 & & 1.887 & 80. & & 79.432 & & 81.85 & \\
\hline $\mathrm{R}_{\text {pred }}^{2}$ & 0.5873 & & .7335 & 0.7 & & 0.7265 & & 0.725 & \\
\hline SEP & 0.379 & & 0.378 & 0.3 & & 0.383 & & 0.382 & \\
\hline $\mathrm{N}_{\mathrm{C}}$ & 3 & & 1 & 1 & & 1 & & 1 & \\
\hline & & & & Field col & tributior & & & & \\
\hline $\mathrm{S}$ & 0.164 & & 0.048 & 0.0 & & - & & 0.044 & \\
\hline $\mathrm{E}$ & 0.257 & & 0.106 & 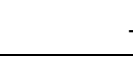 & & 0.102 & & 0.097 & \\
\hline $\mathrm{H}$ & 0.192 & & - & 0.0 & & 0.081 & & 0.077 & \\
\hline $\mathrm{D}$ & - & & 0.135 & 0.1 & & 0.130 & & 0.125 & \\
\hline $\mathrm{A}$ & 0.156 & & 0.097 & 0.0 & & 0.093 & & 0.089 & \\
\hline
\end{tabular}


Table S4. Summary of QSAR results for TR $\beta$ based on superimposition II (the optimal conformation).

\begin{tabular}{|c|c|c|c|c|c|c|c|c|c|}
\hline & CoMFA & \multicolumn{8}{|c|}{ CoMSIA } \\
\hline & $\mathrm{SE}$ & $\mathrm{S}$ & $\mathrm{E}$ & $\mathrm{H}$ & $\mathrm{D}$ & A & SE & $\mathrm{SH}$ & $\mathrm{SD}$ \\
\hline $\mathrm{R}_{\mathrm{cv}}^{2}$ & 0.651 & 0.659 & 0.612 & 0.697 & 0.616 & 0.609 & 0.668 & 0.687 & 0.631 \\
\hline $\mathrm{R}_{\mathrm{ncv}}^{2}$ & 0.676 & 0.668 & 0.656 & 0.938 & 0.658 & 0.651 & 0.674 & 0.934 & 0.677 \\
\hline SEE & 0.341 & 0.345 & 0.352 & 0.156 & 0.350 & 0.354 & 0.342 & 0.160 & 0.341 \\
\hline $\mathrm{F}$ & 83.626 & 80.581 & 76.165 & 139.11 & 77.109 & 74.550 & 82.728 & 144.835 & 83.734 \\
\hline $\mathrm{R}_{\text {pred }}^{2}$ & 0.6090 & 0.6981 & 0.7657 & 0.7801 & 0.7692 & 0.7640 & 0.6222 & 0.7183 & 0.6682 \\
\hline SEP & 0.365 & 0.363 & 0.373 & 0.343 & 0.371 & 0.375 & 0.366 & 0.349 & 0.364 \\
\hline $\mathrm{N}_{\mathrm{C}}$ & 2 & 1 & 1 & 4 & 1 & 1 & 1 & 4 & 1 \\
\hline \multicolumn{10}{|c|}{ Field contribution } \\
\hline $\mathrm{S}$ & 0.105 & 0.116 & - & - & - & - & 0.101 & 0.258 & 0.098 \\
\hline $\mathrm{E}$ & 0.142 & - & 0.149 & - & - & - & 0.134 & - & - \\
\hline $\mathrm{H}$ & - & - & - & 0.730 & - & - & - & 0.501 & - \\
\hline $\mathrm{D}$ & - & - & - & - & 0.171 & - & - & - & 0.154 \\
\hline $\mathrm{A}$ & - & - & - & - & - & 0.143 & - & - & - \\
\hline EEig06d & 0.752 & 0.884 & 0.851 & 0.270 & 0.829 & 0.857 & 0.766 & 0.241 & 0.747 \\
\hline \multicolumn{10}{|c|}{ CoMSIA } \\
\hline & SA & $\mathrm{EH}$ & $\mathrm{ED}$ & EA & $\mathrm{HD}$ & $\mathrm{HA}$ & DA & $\mathrm{SEH}$ & SED \\
\hline $\mathrm{R}_{\mathrm{cv}}^{2}$ & 0.624 & 0.610 & 0.612 & 0.602 & 0.693 & 0.606 & 0.606 & 0.624 & 0.664 \\
\hline $\mathrm{R}_{\mathrm{ncv}}^{2}$ & 0.669 & 0.661 & 0.664 & 0.657 & 0.952 & 0.656 & 0.659 & 0.677 & 0.682 \\
\hline SEE & 0.345 & 0.349 & 0.347 & 0.351 & 0.138 & 0.352 & 0.350 & 0.341 & 0.338 \\
\hline $\mathrm{F}$ & 80.911 & 77.865 & 79.119 & 76.533 & 78.054 & 76.197 & 77.199 & 83.948 & 85.615 \\
\hline $\mathrm{R}_{\text {pred }}^{2}$ & 0.7624 & 0.6269 & 0.7527 & 0.7469 & 0.7595 & 0.7541 & 0.7514 & 0.6017 & 0.5063 \\
\hline SEP & 0.368 & 0.374 & 0.375 & 0.378 & 0.350 & 0.376 & 0.376 & 0.368 & 0.368 \\
\hline $\mathrm{N}_{\mathrm{C}}$ & 1 & 1 & 1 & 1 & 5 & 1 & 1 & 1 & 1 \\
\hline \multicolumn{10}{|c|}{ Field contribution } \\
\hline $\mathrm{S}$ & 0.101 & - & - & - & - & - & - & 0.090 & 0.087 \\
\hline $\mathrm{E}$ & - & 0.131 & 0.126 & 0.130 & - & - & - & 0.119 & 0.115 \\
\hline $\mathrm{H}$ & - & 0.118 & - & - & 0.601 & 0.118 & - & 0.107 & - \\
\hline $\mathrm{D}$ & - & - & 0.149 & - & 0.185 & - & 0.150 & - & 0.136 \\
\hline $\mathrm{A}$ & 0.128 & - & - & 0.124 & - & 0.126 & 0.121 & - & - \\
\hline EEig06d & 0.770 & 0.751 & 0.724 & 0.746 & 0.214 & 0.756 & 0.728 & 0.684 & 0.661 \\
\hline \multicolumn{10}{|c|}{ CoMSIA } \\
\hline & SEA & SHD & SHA & SDA & EHD & EHA & EDA & HDA & SEHD \\
\hline $\mathrm{R}_{\mathrm{cv}}^{2}$ & 0.617 & 0.672 & 0.620 & 0.620 & 0.606 & 0.599 & 0.602 & 0.603 & 0.620 \\
\hline $\mathrm{R}_{\mathrm{ncv}}^{2}$ & 0.674 & 0.936 & 0.672 & 0.676 & 0.669 & 0.661 & 0.664 & 0.664 & 0.685 \\
\hline SEE & 0.342 & 0.157 & 0.343 & 0.341 & 0.345 & 0.349 & 0.346 & 0.348 & 0.336 \\
\hline $\mathrm{F}$ & 82.774 & 135.866 & 82.099 & 83.480 & 80.952 & 78.090 & 79.38 & 78.986 & 86.993 \\
\hline $\mathrm{R}_{\text {pred }}^{2}$ & 0.5831 & 0.7580 & 0.7518 & 0.7511 & 0.7439 & 0.5011 & 0.7354 & 0.7427 & 0.4752 \\
\hline SEP & 0.371 & 0.357 & 0.369 & 0.369 & 0.376 & 0.379 & 0.370 & 0.378 & 0.370 \\
\hline
\end{tabular}




\begin{tabular}{|c|c|c|c|c|c|c|c|c|c|}
\hline $\mathrm{N}_{\mathrm{C}}$ & 1 & 4 & 1 & 1 & 1 & 10 & 1 & 1 & 1 \\
\hline \multicolumn{10}{|c|}{ Field contribution } \\
\hline$S$ & 0.089 & 0.203 & 0.091 & 0.088 & - & - & - & - & 0.079 \\
\hline $\mathrm{E}$ & 0.118 & - & - & - & 0.114 & 0.117 & 0.113 & - & 0.105 \\
\hline $\mathrm{H}$ & - & 0.398 & 0.108 & - & 0.102 & 0.104 & - & 0.102 & 0.094 \\
\hline $\mathrm{D}$ & - & 0.197 & - & 0.137 & 0.134 & - & 0.133 & 0.135 & 0.124 \\
\hline A & 0.113 & - & 0.115 & 0.111 & - & 0.111 & 0.108 & 0.109 & - \\
\hline EEig06d & 0.679 & 0.201 & 0.687 & 0.665 & 0.651 & 0.668 & 0.646 & 0.654 & 0.599 \\
\hline \multicolumn{10}{|c|}{ CoMSIA } \\
\hline & \multicolumn{2}{|l|}{ SEHA } & SEDA & \multicolumn{2}{|c|}{ SHDA } & \multicolumn{2}{|c|}{ EHDA } & \multicolumn{2}{|c|}{ SEHDA } \\
\hline $\mathrm{R}_{\mathrm{cv}}^{2}$ & \multicolumn{2}{|l|}{0.623} & 0.612 & \multicolumn{2}{|c|}{0.616} & \multicolumn{2}{|c|}{0.595} & \multicolumn{2}{|c|}{0.608} \\
\hline $\mathrm{R}_{\mathrm{ncv}}^{2}$ & \multicolumn{2}{|l|}{0.677} & 0.680 & \multicolumn{2}{|c|}{0.680} & \multicolumn{2}{|c|}{0.669} & \multicolumn{2}{|c|}{0.684} \\
\hline SEE & \multicolumn{2}{|l|}{0.341} & 0.339 & \multicolumn{2}{|c|}{0.339} & \multicolumn{2}{|c|}{0.345} & \multicolumn{2}{|c|}{0.337} \\
\hline $\mathrm{F}$ & \multicolumn{2}{|l|}{83.888} & 85.120 & \multicolumn{2}{|c|}{84.835} & 80.666 & & 86.4 & \\
\hline $\mathrm{R}_{\text {pred }}^{2}$ & 0.5881 & & 0.7340 & & & 0.7266 & & 0.72 & \\
\hline SEP & 0.373 & & 0.373 & & & 0.382 & & $0.3^{\prime}$ & \\
\hline $\mathrm{N}_{\mathrm{C}}$ & 1 & & 1 & & & 1 & & 1 & \\
\hline & & & & Field ce & tributio & & & & \\
\hline$S$ & 0.081 & & 0.078 & & & - & & $0.0^{\prime}$ & \\
\hline $\mathrm{E}$ & 0.107 & & 0.104 & & & 0.102 & & 0.0 & \\
\hline $\mathrm{H}$ & 0.096 & & - & & & 0.092 & & 0.0 & \\
\hline $\mathrm{D}$ & - & & 0.123 & & & 0.121 & & 0.1 & \\
\hline $\mathrm{A}$ & 0.102 & & 0.099 & & & 0.098 & & 0.0 & \\
\hline EEig06d & 0.614 & & 0.596 & & & 0.587 & & 0.5 & \\
\hline
\end{tabular}

Table S5. Summary of QSAR results for TR $\alpha$ based on superimposition I .

\begin{tabular}{|c|c|c|c|c|c|c|c|c|}
\hline & CoMF & \multicolumn{7}{|c|}{ CoMSIA } \\
\hline & SE & $\mathrm{S}$ & E & $\mathrm{H}$ & A & SE & SH & SD \\
\hline $\mathbf{R}_{\mathrm{cv}}^{2}$ & 0.526 & 0.375 & 0.388 & 0.282 & 0.305 & 0.532 & 0.313 & 0.445 \\
\hline $\mathrm{R}_{\mathrm{ncv}}^{2}$ & 0.864 & 0.595 & 0.556 & 0.713 & 0.617 & 0.883 & 0.727 & 0.671 \\
\hline SEE & 0.187 & 0.308 & 0.322 & 0.266 & 0.307 & 0.174 & 0.259 & 0.284 \\
\hline $\mathrm{F}$ & 40.291 & 30.851 & 26.258 & 24.806 & 16.096 & 47.698 & 26.591 & 20.418 \\
\hline $\mathrm{R}_{\text {pred }}^{2}$ & 0.6983 & 0.8213 & 0.4972 & 0.7849 & 0.7103 & 0.6222 & 0.8374 & 0.8198 \\
\hline SEP & 0.350 & 0.382 & 0.378 & 0.420 & 0.413 & 0.348 & 0.411 & 0.369 \\
\hline $\mathrm{N}_{\mathrm{C}}$ & 3 & 1 & 1 & 2 & 2 & 3 & 2 & 2 \\
\hline \multicolumn{9}{|c|}{ Field contribution } \\
\hline$S$ & 0.414 & 1.000 & - & - & - & 0.331 & 0.454 & 0.500 \\
\hline $\mathrm{E}$ & 0.586 & - & 1.000 & - & - & 0.669 & - & - \\
\hline $\mathrm{H}$ & - & - & - & 1.000 & - & - & 0.546 & - \\
\hline D & - & - & - & - & & - & - & 0500 \\
\hline
\end{tabular}




\begin{tabular}{|c|c|c|c|c|c|c|c|c|c|}
\hline $\mathrm{A}$ & - & - & - & - & \multicolumn{2}{|c|}{1.000} & - & - & - \\
\hline \multicolumn{10}{|c|}{ CoMSIA } \\
\hline & SA & $\mathrm{EH}$ & ED & EA & HD & HA & DA & SEH & SED \\
\hline $\mathrm{R}_{\mathrm{cv}}^{2}$ & 0.471 & 0.503 & 0.412 & 0.358 & 0.298 & 0.380 & 0.280 & 0.543 & 0.517 \\
\hline $\mathrm{R}_{\mathrm{ncv}}^{2}$ & 0.765 & 0.992 & 0.787 & 0.685 & 0.683 & 0.713 & 0.697 & 0.995 & 0.855 \\
\hline SEE & 0.240 & 0.048 & 0.234 & 0.278 & 0.279 & 0.266 & 0.280 & 0.040 & 0.194 \\
\hline $\mathrm{F}$ & 32.61 & 350.991 & 23.441 & 21.702 & $\begin{array}{c}21.50 \\
4\end{array}$ & 24.809 & 14.541 & 58.396 & 37.255 \\
\hline $\mathrm{R}_{\text {pred }}^{2}$ & 0.8010 & 0.7264 & 0.3251 & 0.3827 & $\begin{array}{c}0.722 \\
1\end{array}$ & 0.7189 & 0.7552 & 0.7370 & 0.5754 \\
\hline SEP & 0.360 & 0.391 & 0.390 & 0.397 & 0.415 & 0.390 & 0.431 & 0.375 & 0.353 \\
\hline $\mathrm{N}_{\mathrm{C}}$ & 2 & 6 & 3 & 2 & 2 & 2 & 3 & 6 & 3 \\
\hline \multicolumn{10}{|c|}{ Field contribution } \\
\hline $\mathrm{S}$ & 0.327 & - & - & - & - & - & - & 0.189 & 0.210 \\
\hline $\mathrm{E}$ & - & 0.575 & 0.640 & 0.579 & - & - & - & 0.506 & 0.486 \\
\hline $\mathrm{H}$ & - & 0.425 & - & - & 0.482 & 0.366 & - & 0.305 & - \\
\hline $\mathrm{D}$ & - & - & 0.360 & - & 0.518 & - & 0.385 & - & 0.305 \\
\hline A & 0.673 & - & - & 0.421 & - & 0.634 & 0.615 & - & - \\
\hline & & & & $\mathrm{Col}$ & & & & & \\
\hline & SEA & SHD & SHA & SDA & EHD & EHA & EDA & HDA & SEHD \\
\hline $\mathrm{R}_{\mathrm{cv}}^{2}$ & 0.470 & 0.371 & 0.451 & 0.435 & 0.416 & 0.402 & 0.328 & 0.343 & 0.471 \\
\hline $\mathrm{R}_{\mathrm{ncv}}^{2}$ & 0.864 & 0.741 & 0.774 & 0.859 & 0.834 & 0.989 & 0.751 & 0.783 & 0.862 \\
\hline SEE & 0.187 & 0.252 & 0.235 & 0.191 & 0.207 & 0.058 & 0.247 & 0.237 & 0.189 \\
\hline$F$ & 40.314 & 28.643 & 34.273 & 38.494 & $\begin{array}{c}31.88 \\
9\end{array}$ & 238.535 & 30.210 & 22.896 & 39.445 \\
\hline $\mathrm{R}_{\text {pred }}^{2}$ & 0.6565 & 0.7880 & 0.7884 & 0.8380 & $\begin{array}{c}0.529 \\
3\end{array}$ & 0.7594 & 0.5042 & 0.7654 & 0.6534 \\
\hline SEP & 0.370 & 0.393 & 0.367 & 0.382 & 0.388 & 0.428 & 0.406 & 0.412 & 0.370 \\
\hline $\mathrm{N}_{\mathrm{C}}$ & 3 & 2 & 2 & 3 & 3 & 6 & 2 & 3 & 3 \\
\hline & & & & Field co & ibution & & & & \\
\hline$S$ & 0.234 & 0.218 & 0.233 & 0.265 & - & - & - & - & 0.157 \\
\hline $\mathrm{E}$ & 0.441 & - & - & - & 0.440 & 0.420 & 0.399 & - & 0.370 \\
\hline $\mathrm{H}$ & - & 0.352 & 0.274 & - & 0.247 & 0.334 & - & 0.276 & 0.195 \\
\hline $\mathrm{D}$ & - & 0.430 & - & 0.250 & 0.312 & - & 0.305 & 0.346 & 0.278 \\
\hline $\mathrm{A}$ & 0.324 & - & 0.493 & 0.485 & - & 0.245 & 0.296 & 0.378 & - \\
\hline & & & & Col & & & & & \\
\hline & SEH & & SEDA & SHL & & EHD & & SEHI & \\
\hline $\mathrm{R}_{\mathrm{cv}}^{2}$ & 0.46 & & 0.445 & 0.4 & & 0.391 & & 0.44 & \\
\hline $\mathrm{R}_{\mathrm{ncv}}^{2}$ & 0.88 & & 0.853 & 0.8 & & 0.824 & & 0.85 & \\
\hline SEE & 0.17 & & 0.195 & 0.2 & & 0.213 & & 0.19 & \\
\hline $\mathrm{F}$ & 48.4 & & 36.873 & 31.3 & & 29.70 & & 36.9 & \\
\hline $\mathrm{R}_{\text {pred }}^{2}$ & $0.69^{\circ}$ & & 0.6631 & 0.81 & & 0.591 & & 0.67 & \\
\hline SEP & 0.37 & & 0.379 & 0.3 & & 0.397 & & 0.37 & \\
\hline
\end{tabular}




\begin{tabular}{|c|c|c|c|c|c|}
\hline $\mathrm{N}_{\mathrm{C}}$ & 3 & 3 & 3 & 3 & 3 \\
\hline \multicolumn{7}{|c|}{ Field contribution } \\
\hline $\mathrm{S}$ & 0.177 & 0.163 & 0.167 & - & 0.126 \\
\hline $\mathrm{E}$ & 0.359 & 0.351 & - & 0.320 & 0.279 \\
\hline $\mathrm{H}$ & 0.199 & - & 0.215 & 0.182 & 0.151 \\
\hline $\mathrm{D}$ & - & 0.230 & 0.300 & 0.263 & 0.235 \\
\hline $\mathrm{A}$ & 0.265 & 0.255 & 0.317 & 0.235 & 0.208 \\
\hline
\end{tabular}

Table S6. Summary of QSAR results for TR $\alpha$ based on superimposition II (the lowest binding

energy).

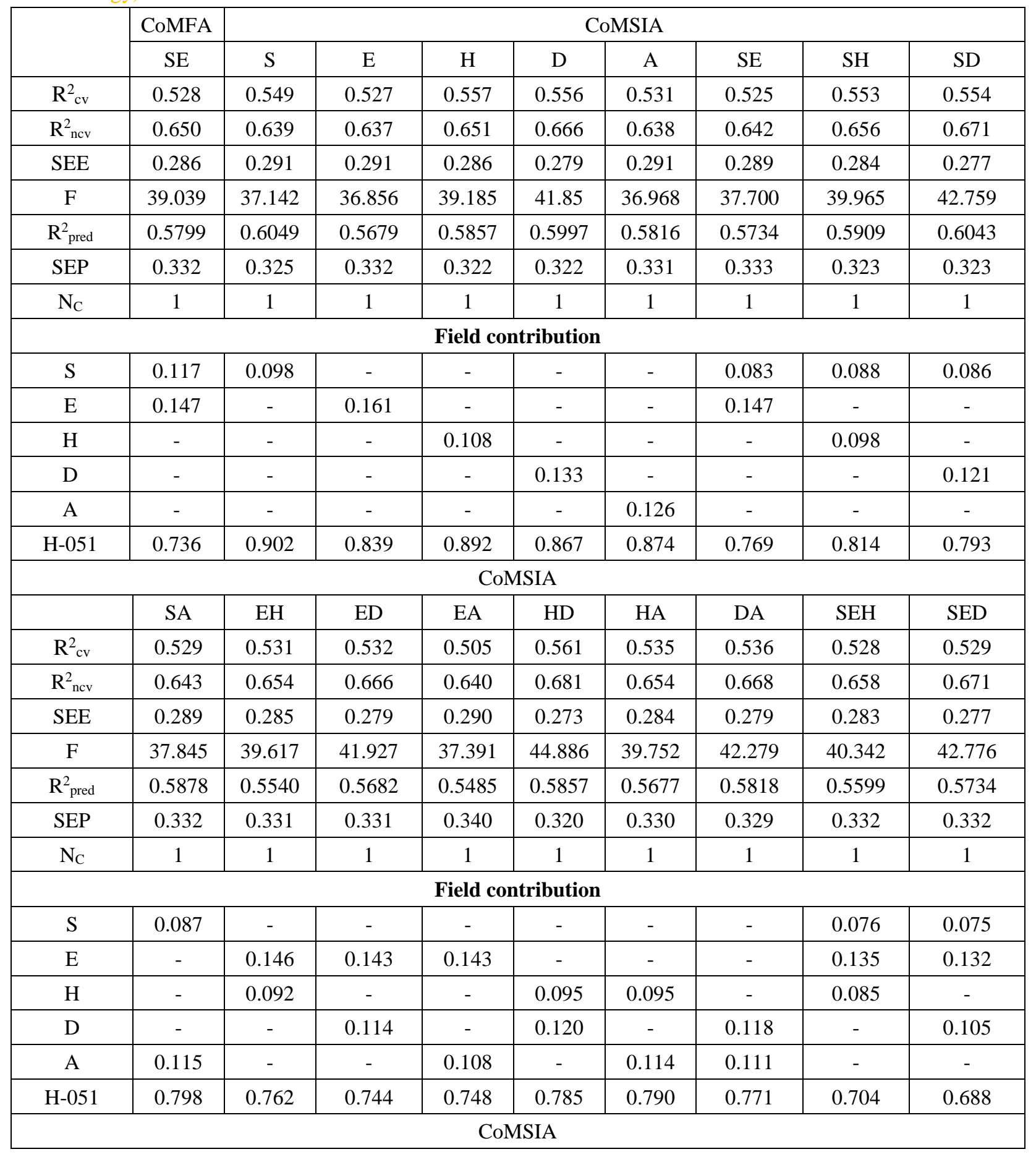




\begin{tabular}{|c|c|c|c|c|c|c|c|c|c|}
\hline & SEA & SHD & SHA & SDA & EHD & EHA & EDA & HDA & SEHD \\
\hline $\mathrm{R}_{\mathrm{cv}}^{2}$ & 0.502 & 0.558 & 0.532 & 0.533 & 0.535 & 0.507 & 0.509 & 0.539 & 0.532 \\
\hline $\mathrm{R}_{\mathrm{ncv}}^{2}$ & 0.645 & 0.685 & 0.659 & 0.673 & 0.681 & 0.656 & 0.668 & 0.682 & 0.684 \\
\hline SEE & 0.288 & 0.271 & 0.282 & 0.277 & 0.273 & 0.284 & 0.279 & 0.272 & 0.272 \\
\hline $\mathrm{F}$ & 38.210 & 45.648 & 40.507 & 43.161 & 44.729 & 39.987 & 42.167 & 45.104 & 45.448 \\
\hline $\mathrm{R}_{\text {pred }}^{2}$ & 0.5546 & 0.5904 & 0.5738 & 0.5878 & 0.5539 & 0.5345 & 0.5475 & 0.5679 & 0.5597 \\
\hline SEP & 0.341 & 0.322 & 0.331 & 0.330 & 0.330 & 0.339 & 0.339 & 0.328 & 0.331 \\
\hline $\mathrm{N}_{\mathrm{C}}$ & 1 & 1 & 1 & 1 & 1 & 1 & 1 & 1 & 1 \\
\hline \multicolumn{10}{|c|}{ Field contribution } \\
\hline $\mathrm{S}$ & 0.075 & 0.079 & 0.079 & 0.077 & - & - & - & - & 0.069 \\
\hline $\mathrm{E}$ & 0.133 & - & - & - & 0.131 & 0.131 & 0.129 & - & 0.122 \\
\hline $\mathrm{H}$ & - & 0.087 & 0.088 & - & 0.082 & 0.083 & - & 0.085 & 0.077 \\
\hline $\mathrm{D}$ & - & 0.111 & - & 0.109 & 0.104 & - & 0.103 & 0.108 & 0.097 \\
\hline $\mathrm{A}$ & 0.100 & - & 0.105 & 0.103 & - & 0.099 & 0.097 & 0.102 & - \\
\hline $\mathrm{H}-051$ & 0.692 & 0.724 & 0.728 & 0.711 & 0.683 & 0.686 & 0.672 & 0.705 & 0.636 \\
\hline \multicolumn{10}{|c|}{ CoMSIA } \\
\hline & \multicolumn{2}{|l|}{ SEHA } & SEDA & \multicolumn{2}{|c|}{ SHDA } & \multicolumn{2}{|c|}{ EHDA } & \multicolumn{2}{|c|}{ SEHDA } \\
\hline $\mathrm{R}_{\mathrm{cv}}^{2}$ & \multicolumn{2}{|l|}{0.505} & 0.506 & \multicolumn{2}{|c|}{0.536} & \multicolumn{2}{|c|}{0.511} & \multicolumn{2}{|c|}{0.508} \\
\hline $\mathrm{R}_{\mathrm{ncv}}^{2}$ & \multicolumn{2}{|l|}{0.660} & 0.672 & \multicolumn{2}{|c|}{0.686} & \multicolumn{2}{|c|}{0.681} & \multicolumn{2}{|c|}{0.684} \\
\hline SEE & \multicolumn{2}{|l|}{0.282} & 0.277 & & & 0.2 & & 0.2 & \\
\hline $\mathrm{F}$ & 40.696 & & 42.998 & 45. & & 44.7 & & 45. & \\
\hline $\mathrm{R}_{\text {pred }}^{2}$ & 0.5409 & & 0.5545 & & & 0.5 & & 0.5 & \\
\hline SEP & 0.340 & & 0.340 & & & 0.3 & & 0.3 & \\
\hline $\mathrm{N}_{\mathrm{C}}$ & 1 & & 1 & & & 1 & & 1 & \\
\hline & & & & Field co & tributior & & & & \\
\hline$S$ & 0.069 & & 0.068 & & & - & & 0.0 & \\
\hline $\mathrm{E}$ & 0.122 & & 0.120 & & & 0.1 & & 0.1 & \\
\hline $\mathrm{H}$ & 0.077 & & - & 0.1 & & 0.0 & & 0.0 & \\
\hline $\mathrm{D}$ & - & & 0.096 & & & 0.0 & & 0.0 & \\
\hline $\mathrm{A}$ & 0.092 & & 0.091 & & & 0.0 & & 0.0 & \\
\hline H-051 & 0.639 & & 0.626 & & & 0.6 & & 0.5 & \\
\hline
\end{tabular}

Table S7. Summary of QSAR results for TR $\alpha$ based on superimposition II (the optimal conformation).

\begin{tabular}{|c|c|c|c|c|c|c|c|c|c|}
\hline & CoMFA & \multicolumn{9}{|c|}{ CoMSIA } \\
\cline { 2 - 10 } & SE & $\mathrm{S}$ & $\mathrm{E}$ & $\mathrm{H}$ & $\mathrm{D}$ & $\mathrm{A}$ & $\mathrm{SE}$ & $\mathrm{SH}$ & $\mathrm{SD}$ \\
\hline $\mathrm{R}^{2}{ }_{\text {cv }}$ & 0.544 & 0.551 & 0.553 & 0.547 & 0.558 & 0.586 & 0.551 & 0.545 & 0.557 \\
\hline $\mathrm{R}_{\text {ncv }}$ & 0.649 & 0.642 & 0.650 & 0.643 & 0.669 & 0.884 & 0.657 & 0.651 & 0.677 \\
\hline $\mathrm{SEE}$ & 0.287 & 0.289 & 0.286 & 0.289 & 0.278 & 0.173 & 0.283 & 0.285 & 0.275 \\
\hline $\mathrm{F}$ & 38.787 & 37.693 & 39.078 & 37.902 & 42.395 & 48.349 & 40.302 & 39.242 & 43.941 \\
\hline $\mathrm{R}^{2}{ }_{\text {pred }}$ & 0.5802 & 0.6053 & 0.5800 & 0.5867 & 0.6003 & 0.5842 & 0.5745 & 0.5906 & 0.6112 \\
\hline $\mathrm{SEP}$ & 0.327 & 0.324 & 0.323 & 0.325 & 0.321 & 0.327 & 0.324 & 0.326 & 0.322 \\
\hline $\mathrm{N}_{\mathrm{C}}$ & 1 & 1 & 1 & 1 & 1 & 3 & 1 & 1 & 1 \\
\hline
\end{tabular}




\begin{tabular}{|c|c|c|c|c|c|c|c|c|c|}
\hline \multicolumn{10}{|c|}{ Field contribution } \\
\hline $\mathrm{S}$ & 0.079 & 0.084 & - & - & - & - & 0.065 & 0.074 & 0.073 \\
\hline $\mathrm{E}$ & 0.207 & - & 0.239 & - & - & - & 0.223 & - & - \\
\hline $\mathrm{H}$ & - & - & - & 0.129 & - & - & - & 0.119 & - \\
\hline $\mathrm{D}$ & - & - & - & - & 0.140 & - & - & - & 0.130 \\
\hline A & - & - & - & - & - & 0.638 & - & - & - \\
\hline $\mathrm{H}-051$ & 0.714 & 0.916 & 0.761 & 0.871 & 0.860 & 0.362 & 0.711 & 0.806 & 0.797 \\
\hline \multicolumn{10}{|c|}{ CoMSIA } \\
\hline & SA & $\mathrm{EH}$ & $\mathrm{ED}$ & EA & $\mathrm{HD}$ & HA & DA & SEH & SED \\
\hline $\mathrm{R}_{\mathrm{cv}}^{2}$ & 0.537 & 0.545 & 0.559 & 0.536 & 0.554 & 0.531 & 0.591 & 0.542 & 0.557 \\
\hline $\mathrm{R}_{\mathrm{ncv}}^{2}$ & 0.644 & 0.656 & 0.682 & 0.932 & 0.678 & 0.643 & 0.875 & 0.662 & 0.688 \\
\hline SEE & 0.288 & 0.284 & 0.273 & 0.136 & 0.274 & 0.289 & 0.179 & 0.281 & 0.270 \\
\hline $\mathrm{F}$ & 37.985 & 40.053 & 45.062 & 61.468 & 44.143 & 37.815 & 44.469 & 41.174 & 46.326 \\
\hline $\mathrm{R}_{\text {pred }}^{2}$ & 0.5901 & 0.5545 & 0.5690 & 0.5505 & 0.5862 & 0.5669 & 0.5946 & 0.5601 & 0.5752 \\
\hline SEP & 0.329 & 0.326 & 0.321 & 0.356 & 0.323 & 0.331 & 0.325 & 0.327 & 0.322 \\
\hline $\mathrm{N}_{\mathrm{C}}$ & 1 & 1 & 1 & 4 & 1 & 1 & 3 & 1 & 1 \\
\hline \multicolumn{10}{|c|}{ Field contribution } \\
\hline $\mathrm{S}$ & 0.069 & - & - & - & - & - & - & 0.059 & 0.059 \\
\hline $\mathrm{E}$ & - & 0.215 & 0.213 & 0.402 & - & - & - & 0.202 & 0.200 \\
\hline $\mathrm{H}$ & - & 0.101 & - & - & 0.113 & 0.106 & - & 0.095 & - \\
\hline $\mathrm{D}$ & - & - & 0.110 & - & 0.124 & - & 0.283 & - & 0.104 \\
\hline $\mathrm{A}$ & 0.184 & - & - & 0.312 & - & 0.177 & 0.350 & - & - \\
\hline $\mathrm{H}-051$ & 0.747 & 0.684 & 0.677 & 0.286 & 0.763 & 0.717 & 0.368 & 0.644 & 0.637 \\
\hline \multicolumn{10}{|c|}{ CoMSIA } \\
\hline & SEA & SHD & SHA & SDA & EHD & EHA & EDA & HDA & SEHD \\
\hline $\mathrm{R}_{\mathrm{cv}}^{2}$ & 0.531 & 0.552 & 0.528 & 0.543 & 0.552 & 0.524 & 0.563 & 0.537 & 0.549 \\
\hline $\mathrm{R}_{\mathrm{ncv}}^{2}$ & 0.652 & 0.684 & 0.649 & 0.676 & 0.686 & 0.650 & 0.933 & 0.675 & 0.692 \\
\hline SEE & 0.285 & 0.272 & 0.286 & 0.275 & 0.271 & 0.286 & 0.136 & 0.276 & 0.268 \\
\hline $\mathrm{F}$ & 39.409 & 45.539 & 38.905 & 43.810 & 45.976 & 38.955 & 62.223 & 43.548 & 47.118 \\
\hline $\mathrm{R}_{\text {pred }}^{2}$ & 0.5552 & 0.5903 & 0.5733 & 0.5897 & 0.5548 & 0.5424 & 0.5553 & 0.5667 & 0.5660 \\
\hline SEP & 0.331 & 0.324 & 0.332 & 0.327 & 0.324 & 0.334 & 0.345 & 0.329 & 0.325 \\
\hline $\mathrm{N}_{\mathrm{C}}$ & 1 & 1 & 1 & 1 & 1 & 1 & 4 & 1 & 1 \\
\hline \multicolumn{10}{|c|}{ Field contribution } \\
\hline$S$ & 0.056 & 0.066 & 0.07962 & 0.061 & - & - & - & - & 0.054 \\
\hline $\mathrm{E}$ & 0.190 & - & - & - & 0.193 & 0.184 & 0.290 & - & 0.183 \\
\hline $\mathrm{H}$ & - & 0.105 & 0.100 & - & 0.091 & 0.087 & - & 0.095 & 0.086 \\
\hline $\mathrm{D}$ & - & 0.116 & - & 0.109 & 0.100 & - & 0.199 & 0.105 & 0.095 \\
\hline A & 0.149 & - & 0.166 & 0.164 & - & 0.144 & 0.231 & 0.158 & - \\
\hline $\mathrm{H}-051$ & 0.605 & 0.713 & 0.673 & 0.666 & 0.615 & 0.585 & 0.281 & 0.642 & 0.582 \\
\hline \multicolumn{10}{|c|}{ CoMSIA } \\
\hline & SEHA & & SEDA & $\mathrm{SH}$ & & $\mathrm{EH}$ & & $\mathrm{SE}$ & \\
\hline $\mathrm{R}_{\mathrm{cv}}^{2}$ & 0.521 & & 0.537 & 0.5 & & 0.5 & & & \\
\hline $\mathrm{R}^{2}{ }_{\mathrm{ncv}}$ & 0.655 & & 0.681 & 0.6 & & 0.6 & & & \\
\hline
\end{tabular}




\begin{tabular}{|c|c|c|c|c|c|}
\hline SEE & 0.284 & 0.273 & 0.273 & 0.274 & 0.272 \\
\hline $\mathrm{F}$ & 39.885 & 44.836 & 44.659 & 44.247 & 45.181 \\
\hline $\mathrm{R}_{\text {pred }}^{2}$ & 0.5431 & 0.5556 & 0.5734 & 0.5346 & 0.5432 \\
\hline $\mathrm{SEP}$ & 0.335 & 0.329 & 0.330 & 0.331 & 0.333 \\
\hline $\mathrm{N}_{\mathrm{C}}$ & 1 & 1 & 1 & 1 & 1 \\
\hline \multicolumn{7}{|c|}{ Field contribution } \\
\hline $\mathrm{S}$ & 0.051 & 0.051 & 0.056 & - & 0.047 \\
\hline $\mathrm{E}$ & 0.174 & 0.173 & - & 0.168 & 0.160 \\
\hline $\mathrm{H}$ & 0.082 & - & 0.090 & 0.079 & 0.075 \\
\hline $\mathrm{D}$ & - & 0.090 & 0.099 & 0.087 & 0.083 \\
\hline $\mathrm{A}$ & 0.137 & 0.136 & 0.150 & 0.132 & 0.126 \\
\hline $\mathrm{H}-051$ & 0.555 & 0.551 & 0.606 & 0.534 & 0.509 \\
\hline
\end{tabular}

$\mathrm{R}^{2}{ }_{\mathrm{cv}}=$ cross-validated correlation coefficient using the leave-one-out methods;

$\mathrm{R}^{2}{ }_{\text {ncv }}=$ Non-cross-validated correlation coefficient; $\mathrm{SEE}=$ Standard error of estimate; $\mathrm{F}=$ Ratio of $\mathrm{R}^{2}{ }_{\text {ncv }}$ explained to unexplained $=\mathrm{R}^{2}{ }_{\text {ncv }} /\left(1-\mathrm{R}^{2}{ }_{\mathrm{ncv}}\right)$;

$\mathrm{R}^{2}{ }_{\text {pred }}=$ Predicted correlation coefficient for the test set of compounds; SEP= Standard error of prediction; $\mathrm{N}_{\mathrm{C}}=$ Optimal number of principal components; $\mathrm{S}=$ steric, E=electrostatic, $\mathrm{H}=$ hydrophobic, $\mathrm{D}=\mathrm{H}-$ bond donor, $\mathrm{A}=\mathrm{H}$-bond acceptor.
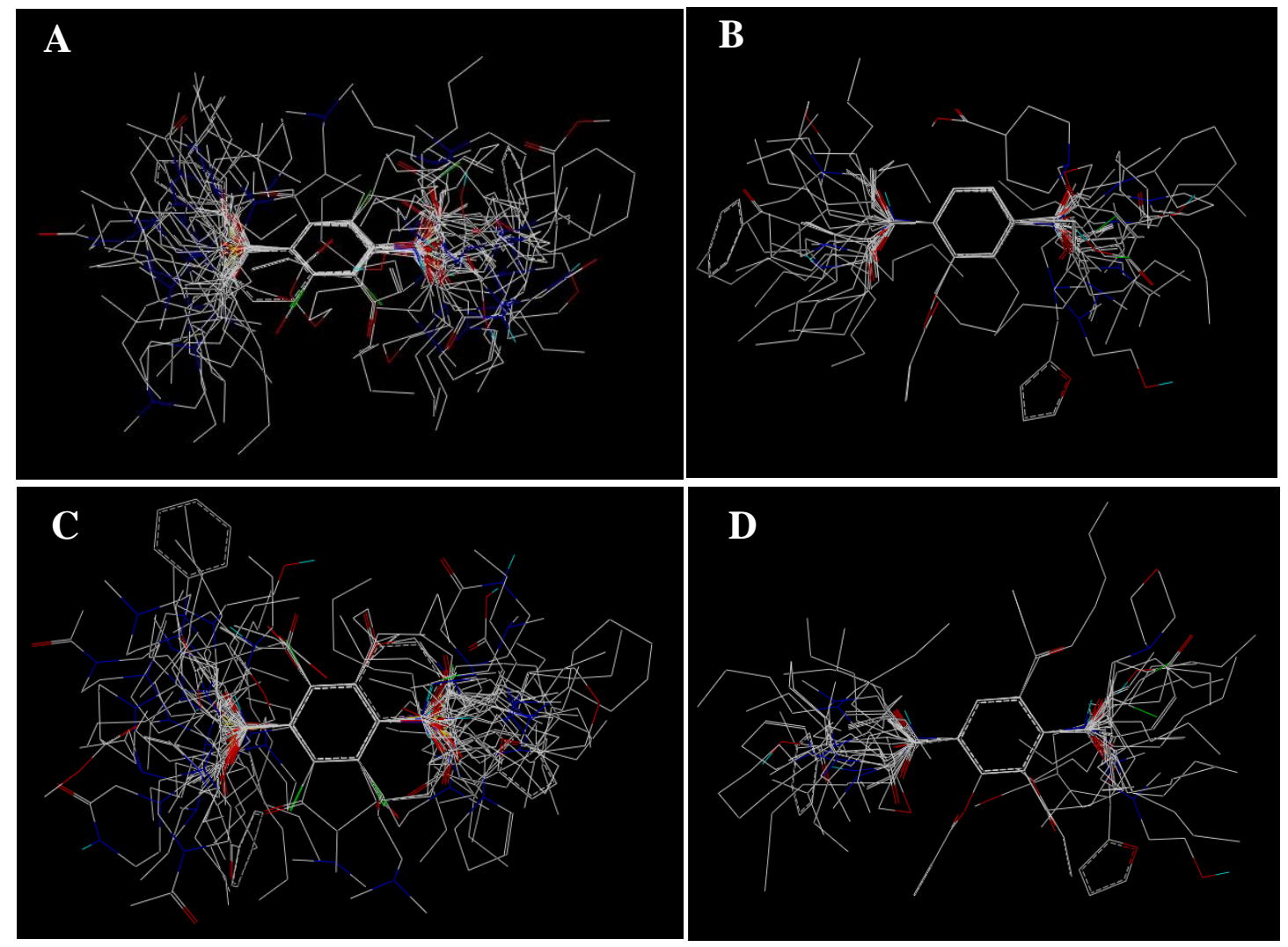

Figure S1 (A) Present the alignments for TR $\beta$ from the superimposition II based on the lowest

binding energy; (B) Present the alignments for TR $\alpha$ from the superimposition II based on the 
lowest binding energy; (C) Present the alignments for TR $\beta$ from the superimposition II based on the optimal conformation; (D) Present the alignments for TR $\alpha$ from the superimposition II based on the optimal conformation. 